\title{
Global assessment of water challenges under uncertainty in water scarcity projections
}

\author{
P. Greve*1, T. Kahil ${ }^{1}$, J. Mochizuki ${ }^{1}$, T. Schinko ${ }^{1}$, Y. Satoh ${ }^{1}$, P. Burek ${ }^{1}$, G.

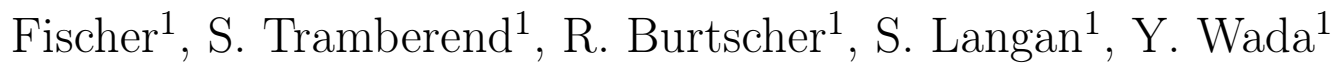 \\ ${ }^{1}$ International Institute for Applied Systems Analysis, Laxenburg, Austria
}

August 2, 2018

\begin{abstract}
Water scarcity, a critical environmental issue worldwide, has primarily been driven by a significant increase in water extractions during the last century. In the coming decades, climate and societal changes are projected to further exacerbate water scarcity in many regions worldwide. Today, a major issue for the ongoing policy debate is to identify interventions able to address water scarcity challenges in the presence of large uncertainties. Here, we take a probabilistic approach to assess global water scarcity projections following feasible combinations of Shared Socioeconomic Pathways (SSPs) and Representative Concentration Pathways (RCPs) for the first half of the 21st century. We identify - alongside trends in median water scarcity - changes in the uncertainty range of anticipated water scarcity conditions. Our results show that median water scarcity and the associated range of uncertainty are generally on the increase worldwide, including many major river basins. Based on these results, we develop a general decision-making framework to enhance policymaking by identifying four representative clusters of specific water-policy challenges and needs.
\end{abstract}

A reliable and sufficient availability of water is one of the key factors addressed by the Sustainable Development Goals (SDGs) agreed by the United Nations in 2015². Most of the 17 SDGs are affected, either directly or indirectly, by growing water scarcity problems ${ }^{2}$. Significant increases in water withdrawals throughout the 20th century and during the first decades of this century ${ }^{3}$ have led to severe water scarcity in many regions of the world ${ }^{4,5}$ and changes in mean hydro-climatological conditions under climate change ${ }^{6-8}$ potentially exacerbate water scarcity in those regions. Integrated assessments considering both socio-economic scenarios as represented within the Shared Socioeconomic Pathways (SSPs) and climate change scenarios driven by the Representative Concentration Pathways (RCPs) both at regional ${ }^{9,10}$ and global scales ${ }^{5,11-14}$ are

*greve@iiasa.ac.at 
thus crucial in terms of identifying policy interventions to address water scarcity locally and in a regional to global context. A wealth of studies with a global focus ${ }^{14-17}$ provide general management options to address water scarcity, including among many others, investments in water storage and transfer infrastructures, desalination plants, efficient irrigation systems, improved crop water productivities, and/or water trade and economic incentives, informing much needed localized adaptation assessments ${ }^{9,10,18-22}$. However, it is important to acknowledge here that local assessments cannot be substituted for, but potentially framed and guided through global analyses.

Decisions on investments and interventions at all scales should be based on reliable projections of future water scarcity conditions. However, the wide range of anticipated socioeconomic developments and methods ${ }^{23,24}$, greatly intensify the uncertainty in projections of future water supply and demand. A comprehensive assessment of i) underlying uncertainties in water scarcity projections; and ii) how these uncertainties impact the planning and decision-making process on a regional to global scale is currently lacking. Here, we aim to address this gap by analyzing the wide range of possible future conditions likely to pose significant planning challenges for water management authorities in vulnerable world regions and river basins. Decisions on investments required in infrastructure and other system improvements are complex and thus associated with high risks of maladaptation and large costs of failed investments. Moreover, decisions taken could ultimately lead to the degradation of valuable assets such as non-renewable groundwater resources ${ }^{25}$ and/or water-dependent ecosystems ${ }^{26}$.

In this study, we assess global water challenges arising from i) anticipated median changes in water scarcity conditions, in combination with ii) the associated uncertainty in water scarcity projections. The assessment uses a multi-model ensemble that enables us to establish a comprehensive and innovative policy framework as a basis to frame decision making at regional to global scales. While not substituting the need for place-specific assessments and dialogs, the normative nature of this study makes a critical contribution to the future processes involved in planning and implementing no-regret, transitional and transformational investment options by governmental, private-sector and civil society actors.

\section{Data and Approach}

To assess the uncertainty, we use an ensemble of 45 future water scarcity projections based on i) three global hydrological models (GHMs); ii) five climate models (GCMs); and iii) three water scenarios. The GHMs represent a comprehensive collection of state-of-the-art hydrological models designed to capture both water supply and human water demand at grid level. The GHMs are forced using five, bias-corrected, state-of-the-art GCMs from the Coupled Model Intercomparison Project Phase 5 (CMIP5) initiative ${ }^{27}$. The model runs were performed under three water scenarios based on feasible combinations of Shared Socio-Economic Pathways (SSPs) and different Representative Concentration Pathways (RCPs) and developed under the Water Futures and Solutions (WFaS) Initiative of the International Institute for Applied Sys- 
tems Analysis (IIASA) ${ }^{14}$. The scenarios comprise (i) a Sustainability scenario (SSP1-RCP4.5), (ii) a Middle-of-the-Road scenario (SSP2-RCP6.0) and (iii) a Regional Rivalry scenario (SSP3RCP6.0).

The data used is provided on a $0.5^{\circ} \times 0.5^{\circ}$ global grid and for five decadal climatologies within the first half of the 21st century (2006-2015 to 2046-2055). We use model estimates of both water supply (i.e., available water resources) and water demand (i.e., water withdrawal), which are both directly and indirectly influenced by climatic changes and socio-economic developments. We further aggregate water demand and supply over a set of large rivers to illustrate our results at basin-scale. However, it is important to consider that moving along scales (such as e.g. from grid cell to basin-scale) could potentially affect the results and their associated interpretation $^{28,29}$. To assess changes in water scarcity, we compute the ratio of the decadal averages of water demand to water supply, here referred to as the water scarcity index (WSI), at each grid cell. A WSI of $0.2-0.4$ is interpreted as mild or emerging water scarcity conditions. We use WSI $\geq 0.4$ to indicate conditions under which human water use accounts for about $40 \%$ or more of the average water supply - usually denoted as (severely) water scarce ${ }^{30}$. Water scarcity thresholds and their associated uncertainties have been assessed previously ${ }^{31,32}$. Uncertainty is quantified in terms of the interquartile range (IQR, range between the 25th and 75th quantile). The multi-forcing, multi-model and multi-scenario ensemble also allows us to attribute the dominant drivers of uncertainty. The uncertainty attribution is based on previously utilized approaches $^{33}$ and we assess the fraction of uncertainty that each source (related to the choice of GHM, GCM or scenario) contributes to the total uncertainty (for all land area with data). This approach provides basic information on the relative importance of different uncertainty sources while it, however, neglects any potential dependencies between the individual sources of uncertainty by assuming each source of uncertainty to be independent.

In order to simplify the complex output of the model ensemble, to assess the potential policy implications and to assist decision making, we further classify areas according to i) their median level of WSI in 2050 (severe or non-severe); ii) their initial level of uncertainty (IQR) in 2010; and iii) the decade-to-decade evolution of IQR between 2010 to 2050, following a clustering approach based on local optimization. Please refer to the Methods section for more information on the data and the methodological approaches

Additionally, even though this study is not intended to address impacts of seasonal water scarcity, we acknowledge that water scarcity might be an important issue at seasonal scales and especially within months of low water supply ${ }^{13,34,35}$. Hence, we include supplementary material showing an assessment of WSI for the month with the lowest water supply, which will be addressed in the assessment of the policy implications.

\section{Uncertainty in water scarcity projections}

The multi-model and multi-scenario ensemble allows us to comprehensively assess changes in median WSI and the IQR for the first (1: 2006-2015) period and the associated change until 
the last period (5: 2046-2055, Figure 1, please also see Supplementary Figure 1). Severe water scarcity conditions $(W S I>0.4)$ within the first period are primarily found in regions such as Pakistan, Central Asia, Eastern China, and the mid-western United States. IQR is additionally high in much larger regions, including parts of southern Africa, the Mediterranean region, the Arabian Peninsula, and Australia.

We further assess the relative increase in the median and IQR between the first and last decade of our study period (Figure 1c,d). Median WSI is generally growing with a relative increase of up to $100 \%$ in parts of northern Africa, the Middle-East, and East Asia. This change is widely associated with a similar relative increase in the IQR, also showing a doubling (and higher) in many parts of, for example, central North America, South Asia, and the Mediterranean region, thereby indicating that the future development of water scarcity is highly uncertain.

The relative importance of uncertainty stemming from the different sources within the 20462055 time period is illustrated in Figure 2. The figure clearly shows that in the majority of regions, most of the overall uncertainty is generated by the choice of GHM. In some regions, however, the choice of GCM also significantly contributes to the overall uncertainty, especially in parts of India and many subtropical monsoon regions in particular. Uncertainties originating from the choice of scenario are in most cases superimposed by those of other drivers but reach quite high values in parts of, for example, Central Europe or the Eastern United States.

By focusing on a subset of major river basins, Figure 3 further illustrates i) the complex concurrent behavior of both the change in the median response and the change in uncertainty; and ii) the time-evolution of the relative importance of different sources of uncertainty. For the Yangtze Basin there is, for example, little change in the overall uncertainty, but nearly a constant increase in WSI across all quantiles, surpassing the indicated threshold for severe water scarcity in the higher quantiles from the 2030s onwards. For other basins such as, for example, the Danube, Syr Darya, and Tigris-Euphrates, the lowest quantiles do not show changes in WSI, while the higher quantiles show increases, pointing toward an increased risk of experiencing severe water-scarce conditions. It is, however, important to note that there are strong spatial variations within the particular basin areas and that aggregation of hydroclimatogical data might be subject to rescaling issues ${ }^{28,29}$. Uncertainties stemming from the choice of GHM dominate throughout the study period, partly showing slight decreases due to growing scenariospecific uncertainty. GCM-related uncertainty is particularly high in South-Asian basins.

\section{Water challenges under uncertainty}

The dynamic developments in water scarcity projections presented here could have significant bearing on regional policymaking. The water-sector challenges may differ considerably for those countries and regions facing different levels of baseline WSI under future uncertainty. Incremental improvements in water-use efficiency, for example, may suffice for those areas with a marginal (and relatively certain) increase in projected WSI. For those areas facing severe water scarcity now and high uncertainty in the future, however, longer-term planning could 
pose a particular challenge ${ }^{36}$.

To help understand the potential policy implications of these findings and guide the planning for large-scale adaptation strategies in the coming decades, we present a novel typology of global water scarcity challenges. Figure 4 defines four clusters (or challenge classes), highlighting areas with potentially similar water management challenges in each (please also refer Supplementary Figure 2 for zoomed-in map segments of most affected regions). By using a clustering approach we intend to simplify the complexity that is inherent to an ensemble of sophisticated model output. We acknowledge that water management is constrained by local conditions and there might be few global solutions to local water management challenges. However, based on previous studies assessing impacts and policy-making under uncertainties of water supply ${ }^{37}$, the typology developed in this study aims to provide a framework to help shape the future research agenda, global investments, and regional policymaking related to the different challenges of water scarcity under uncertain future projections and the type of policy interventions (e.g., soft vs. hard path, transitional vs. transformational) that could be used. This also implies that this topology does not aim to substitute the need for regional and local assessments of water solutions.

\section{Low challenge area (blue)}

Characterized by WSI $<0.4$ in 2050 with relatively low and constant uncertainties (i.e., generally also those simulations projecting relatively high WSI will likely not include severe water-scarce conditions). These regions are primarily located in Central Europe, Turkey, eastern India and Southeast and East Asia including Japan. In these regions, the current practice of water supply and use would likely continue to be used in the future. However, the need for (additional) actions should be evaluated regularly; facing acute water scarcity during a certain period of the year when water demand is extremely large or during drought periods when water availability is exceptionally reduced, cannot be ruled out, especially at local scales. In above $80 \%$ of the total area within this cluster, severe water scarcity potentially occurs within months of lowest water supply (see also Supplementary Figure 3). Hence, regular monitoring activities and risk reevaluations are suggested, both long-term and seasonal. These regions may follow conventional decision-making approaches such as, for example, cost-benefit-analysis (CBA).

\section{Medium challenge area (green)}

Characterized by WSI $<0.4$ in 2050, although with increasing uncertainty, including the risk of severe water scarcity in some model runs. These regions are located primarily in eastern Europe (including parts of Russia), central USA, parts of Africa, Northeast Brazil, and parts of the Middle East. While water scarcity is not imminent in all model runs performed, the potential impacts of unexpected scarcity possibly place a large burden on society. Therefore, these regions may start by implementing what is classified as no-regret (soft-path) transitional options that yield benefits even if projections are proven to be highly uncertain. These are 
improvements in water-use efficiency through, for example, the adoption of water-saving equipment in households and industries or the use of pressurized systems for irrigation ${ }^{38}$. Additional management options that could be implemented without incurring large upfront costs include optimized operation and maintenance of existing infrastructure which lead to reduced nonrevenue water and improved crop water productivity with the help of new cultivars or higher efficiency of nutrient application ${ }^{39}$. There are further opportunities to change water-use behavior in these regions through the use of well-designed water-tariff structures that reflect the full cost of water supply as well as the scarcity value of the resource, engagement with stakeholders, education, and the use of social norms. Given that projected levels of WSI are not extremely high (even in the upper-range projection), these transitional options may effectively address the projected water scarcity conditions. At the same time, in order to take the potential risk of high water scarcity into account (as projected in the upper range of the uncertainty distribution), these regions may also need to implement risk-reduction strategies such as the establishment of adequate monitoring and early warning systems, more flexible operational rules for water facilities, conjunctive use of alternative water sources, diversification of agricultural production, and the use of crop insurance and compensation schemes. Similar to the low-challenged areas, in more than $80 \%$ of the affected area, severe water scarcity potentially occurs within months of lowest water supply (see Supplementary Figure 3), requiring additional actions to overcome seasonal water scarcity. Effective decision making may follow iterative and/or robust processes in these regions ${ }^{40}$.

\section{Medium challenge area (yellow)}

Characterized by WSI $>0.4$ in 2050 with a relatively constant range of uncertainty (i.e., low- and upper-range projections will likely be relatively close to the projected median). These regions are primarily located in Central Asia, northern China, and South India, including some major cities also elsewhere as shown in Supplementary Figure 2. Given the high WSI projected by the majority of the model ensemble, immediate action is necessary to change the current practice of water supply and use in these regions. Potential policy interventions may include not only transitional changes, as in the green medium challenge area, but also transformational changes (hard-path). While distinctions between transitional and transformational changes remain open to debate, transformation, in general, entails a change in the fundamental attributes of natural and human systems ${ }^{41}$. In this case, transformational changes cover investments in large water infrastructure and advanced technologies, including storage facilities such as dams; water transfer; water recycling and reuse; and where viable, desalination. Transformational change may also include planned relocation of industry, streamlining of business activities, and development of alternative livelihoods ${ }^{21,22,42}$.

Transformational changes have been observed in response to the recent drought, for example, in the Murray-Darling Basin of Australia where thousands of irrigators have voluntarily sold their water entitlements to the environmental buy-back program. As large cutbacks in entitlements have substantially reduced farmers' revenues, most irrigators have abandoned farming activities 
altogether or have converted to dryland farming ${ }^{43}$.

Another example is Singapore, where the population faced acute water scarcity and dependence on imported water from Malaysia, and subsequently implemented a major change, transforming the water-resources system into one based on non-conventional sources, such as rainwater harvesting, desalination, and sophisticated technologies for collecting and recycling wastewater ${ }^{18}$.

\section{High challenge area (red)}

Characterized by WSI $>0.4$ in 2050 with high and increasing uncertainties in the projections (i.e., extreme projections could be very different from the median response). These regions are primarily located in parts of the southern USA and Mexico, northern Africa, parts of the MiddleEast, northern China, as well as many parts of Northwest India and Pakistan. Given that the projected WSI is high in most model runs, transitional changes will unlikely suffice in most cases. These regions must go beyond considering conventional low-regret policy options and pay particular attention to the need for transformational changes. While large-scale interventions such as dams and water transfer are often costly and irreversible, planning for these investments poses a particular challenge in this area due to high and increasing uncertainty. To avoid lock-in and maladaptation, such investments must be carefully researched and designed before commitments are made. In certain cases, modular options that allow for additions and reversals may be more advisable, as, also, may be options such as green infrastructure that have lower environmental impacts and hence reduced risks of lock-in ${ }^{44}$. Rethinking the ways in which water is allocated to societal needs is also a major step toward achieving transformational changes. As a context-specific approach, in countries such as Chile, Australia, and the USA, for example, recent decades have seen a major shift toward market-based water-allocation systems with varying degrees of success ${ }^{45}$. For instance, the emergence of a water market in the Murray Darling Basin of Australia, and the ensuing flexibility in water reallocation allowed the gross value of irrigated agricultural production to remain virtually unchanged during the recent drought, despite a fall in extractions of about $70 \%$ from 2000 to $2008^{46}$. However, the evidence from the Murray-Darling basin indicates that additional measures need to be implemented to protect against the potential third-party effects of market-based water allocation such as reduced streamflow and increased groundwater pumping ${ }^{47}$.

The concept of virtual water trade also offers a lens through which to analyze and transform global trade patterns. By taking proper account of hidden water transfer embedded in traded goods, a country may decide to improve local water scarcity through imports of water-intensive products from areas with abundant water resources ${ }^{25,48}$. For instance, food imports have been used to overcome local limits to growth, affecting three billion people worldwide - or $81 \%$ of the population that is approaching or has already exceeded local carrying capacity. Lifestyle changes, such as reduction in food waste and meat consumption, when aggregated at the larger scale, may also have transformative impacts ${ }^{49}$. Further research will be of additional assistance in reducing model uncertainty in these regions. 


\section{Discussion}

The impact of future climatic and socioeconomic changes on the water sector in the different cluster areas could be tackled by the adaptation policies mentioned above. However, implementation of these policies is challenged by governance structure, socio-political and financial barriers, and the lack of capacity to implement changes. Adaptation policies can deliver expected outcomes only when they are backed by robust institutional infrastructure, enhanced local capacities, and improved water governance ${ }^{50}$. Furthermore, massive investments in grey water infrastructure may trigger harmful environmental impacts on river tributaries and could risk loss of capacity to sustain human activities and ecosystem functioning. Infrastructure further increases the risk of conflicts over water resources such as, for example, upstream vs. downstream relations. Integrative planning must be applied in areas where investments are needed, with the goal of finding a balance between tapping economic potential and sustaining key natural ecosystems ${ }^{20}$. Lastly, adaptation is a challenging task for regions with limited economic capacity to invest in capital-intensive water infrastructure. Well-designed policies are needed to overcome these barriers, including a better alignment of policies across water-related domains which would reduce overall investment costs, and the use of adequate incentives to mitigate risks associated with long-term water investment projects so as to foster both local and foreign investments ${ }^{44}$. We do further recognize that water challenges of any kind could also appear at low WSI and hence also outside the clustered regions.

In addition to the level and the changes in uncertainty over time, also the sources of uncertainty may have an influence on policy and decision making. If the main source of uncertainty is the choice of a water scenario, this is a strong and clear call for fostering climate change mitigation and adaptation in order to tackle the RCP signal and/ or to mainstream risk-sensitive socioeconomic development in order to address the SSP signal. Since in our assessment the largest contributions to uncertainty stem for most of the world from either GHMs or GCMs, it will be paramount to invest in improving information gathering techniques in addition to the application of iterative and robust decision making processes. More sophisticated observation and measurement techniques as well as modeling approaches (e.g. by a better representation of physical and socioeconomic processes) may help to bring uncertainties down over time and to ensure confidence in long-term policy planning.

Our results attest to the importance of changes in median WSI in the model ensemble projections as well as the associated uncertainties. This approach provides a new perspective on water challenges under changing water scarcity conditions that go beyond the wide-range of possible solutions presented in previous assessments ${ }^{14-17}$. Effective policymaking will recognize the wide range of implications arising from large uncertainties and risks in future projections. Evaluating alternative scenarios beyond the median projection thus helps avoid maladaptation, adverse path dependencies, and the large costs of error. The results of this global study call for a careful and deliberative design of water-policy interventions, especially in the mediumto-high challenge areas identified, that could potentially also help in the context of achieving 
the SDGs. As new scientific knowledge, empirical insights, and breakthroughs in technological options become available, this assessment should be conducted iteratively to reflect these latest developments. For the countries with high uncertainty, as outlined above, policies should be designed to be robust under a wide range of socioeconomic and climate conditions, and able to adapt to conditions that both can and cannot be anticipated. For areas with potential needs for transformational changes, however, a mere focus on robustness may not be sufficient. More fundamental transformations may be required to address severe water scarcity problems.

However, it is also important to note that the range and distribution of model runs within the multi-model ensemble is highly variant between grid points. Breaking down this information into four classes involves a certain degree of subjectivity and fuzziness (e.g. also through the choice of water scarcity thresholds being applicable at different scales of analysis ${ }^{28,29}$ ), and thus does not permit us to evaluate our results beyond the generic management interventions presented here. How these clusters are, for example, sensitive to the choice of the water scarcity threshold (here WSI = 0.4) is further explored in Supplementary Figure 4. It is also important to note that the results presented here are not primarily intended to be interpreted at the grid point level, but should rather help the identification of contiguous areas and hotspots facing similar water challenges, also at sub- to multi-basin and within-country scales. However, at the same time our results can help to identify small-scale hotspots, such as e.g. individual major cities, facing distinct challenges in comparison to surrounding areas, thereby providing a major advantage over basin- or country-scale assessments.

The four challenge classes could help to facilitate policymaking in the respective areas as they take up the adaptation challenge and develop strategies to reduce water-system vulnerability to future climatic and socioeconomic changes, but are by no means a substitute for an indepth investigation of context-specific policies and management options. Hence, this study could initiate and support local-to-regional assessments and case studies aiming to evaluate the potential of specific management options under uncertainty in the anticipated conditions and could be used as a starting point for shaping future research agenda and policy discourses on this topic. Even though we acknowledge that it is challenging to connect global scale models to regional water management practices, it is our assessment that, despite the uncertain nature of the models, providing possible avenues for better water management planning at largescale strategic levels and in the context of uncertainties is potentially useful for regional water managers. 


\section{Methods}

\section{Data overview}

We use an ensemble of 45 future-water scarcity projections based on i) three GHMs; ii) five climate models (GCMs); and iii) three water scenarios. The three state-of-the-art GHMs used are: i) PCR-GLOBWB ${ }^{51,52}$, (ii) $\mathrm{H} 08^{53}$, (iii) WaterGAP2.2 $2^{54,55}$ and compute both water supply and human water use at grid level. As water supply, we consider here both local runoff and water inflow from neighboring grid cells. Water use is computed as the sum of domestic and industrial water demand and water used for irrigation. The models are process-based, featuring e.g. surface and subsurface runoff, river discharge, groundwater recharge, and water storage in soils, snow, the canopy, groundwater, surface water bodies and wetlands. These hydrological processes are computed at daily scale, with storage acting also on longer time scales (e.g., days to decades such as groundwater). Available water is supplied to a grid cell either as local runoff (surface, subsurface, and baseflow) or inflow from neighboring grid cells along river networks based on realistic routing schemes. Soil hydrology is represented by moisture storage and exchange between several soil layers as well as water exchange between the upper soil and the underlying groundwater reservoirs. Water exchange between soils and the atmosphere (soil and canopy evaporation, transpiration) and from surface water bodies are parameterized as well. Changes in water demand are computed for three different sectors: domestic and industrial water use and irrigation. Three models assess domestic and industrial water use as a function of e.g. GDP and population depending on the different future water use scenarios (based on feasible RCP-SSP combinations) ${ }^{14}$. Irrigation water demand is computed using meteorological conditions, crop intensity, and area and irrigation efficiency assumptions. Water withdrawals are originating either from surface water bodies (rivers, lakes, reservoirs), from groundwater or from other sources (such as e.g. desalination in PCR-GLOBWB). In addition, the WaterGAP model, has been calibrated against observed river discharge for better regional water balance assessments, while the other two models have been extensively validated at the global scale for different model outputs, such as e.g. (local) runoff, river discharge, groundwater, soil moisture, evapotranspiration, and water demand. The GHMs are forced by using five state-of-the-art GCMs (HADGEM2-ES, IPSL-CM5A-LR, MIROC-ESM-CHEM, GFDL-ESM2M, NORESM1M) from the Coupled Model Intercomparison Project Phase 5 (CMIP5) ${ }^{27}$ initiative that were bias-corrected and utilized within the Inter-Sectoral Impact Model Intercomparison Project (ISI-MIP) fast track initiative ${ }^{56}$. The model runs were performed under three water scenarios based on the feasible combinations of Shared Socio-Economic Pathways (SSPs) and different Representative Concentration Pathways (RCPs). Within these scenarios, climate-change forcing is represented by the RCP scenarios and correspond to relative radiative forcing reached by the end of the 21st century in comparison with the preindustrial period. These are 2.6, 4.5, 6.0, and $8.5 \mathrm{Wm}^{-2}$ and the respective pathways are usually referred to as RCP2.6, RCP4.5, RCP6.0 and RCP $8.5^{23}$. Socio-economic projections are represented within the SSP-scenarios ${ }^{24}$, which project important policy drivers including, for example, population growth and Gross Domestic 
Product (GDP) within five different scenarios (SSP1 - SSP5). However, the SSP-scenarios do not specifically focus on water-related issues. Therefore, within the WFaS Initiative the basic SSP-pathways were extended at a country-level to better account for water availability and water demand from all relevant sectors. Here, we use three water scenarios based on feasible combinations of RCPs and $\operatorname{SSPs}^{14}$. These are (i) a Sustainability scenario (SSP1-RCP4.5), (ii) a Middle-of-the-Road scenario (SSP2-RCP6.0) and (iii) a Regional Rivalry scenario (SSP3RCP6.0).

\section{Masking non-water scarce regions}

Non-water scarce regions are masked by excluding all grid points where the 75th quantile of all water scarcity projections is below 0.1 at all time steps - this is to exclude regions that are highly unlikely to be under water stress even in the most pessimistic ensemble simulations. Additionally, grid cells with very low average water demand $\left(<1.000 \mathrm{~m}^{3} /\right.$ day $)$ are neglected, thereby also indirectly excluding the very water-scarce and unpopulated regions (please see Supplementary Figure 1 for raw results).

\section{Assessing sources of uncertainty}

We assess the fraction of uncertainty that each source (related to the choice of GHM, GCM, or scenario) contributes to the total uncertainty at grid point level ${ }^{33}$. We compare i) the average over the variances of all GCMs under each water scenario and for each GHM (GCM uncertainty); ii) the average of the scenario-specific variances of the averages over all GCMs for each GHM (GHM uncertainty); and iii) the variance of the averages of all GHMs and GCMs within a specific scenario (scenario uncertainty). However, this approach neglects any potential dependencies between the individual sources of uncertainty. Please also note that any uncertainty stemming from the choice of scenarios is actually intentional, whereas uncertainties from GCMs and GHMs are due to model deficiencies.

\section{Cluster approach}

In a first step, we determine different classes of uncertainty characteristics following a clustering approach based on local optimization against the initial IQR and decade-to-decade changes in IQR at grid point-level. This allows us to separate land areas into regions of low/high and stable/increasing uncertainty. In a next step, we further determine if these areas are located in severely water scarce regions by 2050 regarding the median response.

Clustering is performed at grid point level using two different characteristics of the ensemble of water scarcity projections. These are: i) the initial level of uncertainty (characterized by the IQR) in 2010; and ii) the decade-to-decade changes in IQR between 2010 to 2050 (resulting in 4 estimates: changes between (1) 2010 to 2020, (2) 2020 to 2030, (3) 2030 to 2040 and (4) 2040 to 2050). Due to the exploratory nature of clustering approaches, a local optimization (k-means) approach was first used by applying a set of distance functions/metrics in order to determine 
the optimal and most meaningful number of clusters. The clustering was performed under Mathematica 9.0 (Wolfram Research, Inc., Mathematica, Version 9.0, Champaign, IL, 2012). Since each grid point hence consists of five numerical characteristics (representing basically a vector), we used here besides a Squared Euclidean distance, $d(p, q)=\sum_{i=1}^{n}\left(q_{i}-p_{i}\right)^{2}$, also metrics such as the Manhattan, $d(p, q)=\sum_{i=1}^{n}\left|q_{i}-p_{i}\right|$, and the Canberra distance, $d(p, q)=$ $\sum_{i=1}^{n}\left|q_{i}-p_{i}\right| /\left(\left|p_{i}\right|+\left|q_{i}\right|\right)$, with $p$ and $q$ representing arbitrary vectors. The clusters presented in this study are robust among the different metrics and the number of clusters, always partitioning the data into regions of low or high initial uncertainty and either no/small or medium to large changes in uncertainty. By taking into account the projected level of the WSI for the mid-century time period, we selected four classes that are represented through distinct management challenges - (i) low challenge - blue: WSI $<0.4$, rel. small IQR, stable IQR, (ii) medium challenge - green: WSI $<0.4$, rel. small/medium IQR, increase in IQR, (iii) medium challenge - yellow: WSI $>0.4$, rel. small/medium IQR, stable IQR, (iv) high challenge red: WSI > 0.4, medium/large IQR, increase in IQR. To avoid spurious and inconsistent results for isolated grid points (those with all neighboring grid points masked out), these are omitted from the analysis (please see Supplementary Figure 5 for comparison). The results presented here are finally based on the Canberra metric.

Data availability The WFaS data that support the findings of this study are available from the corresponding author upon request.

Author Information Correspondence and requests for materials should be addressed to P.G. (greve@iiasa.ac.at). The authors declare no competing financial interests.

Acknowledgments The authors acknowledge the Global Environment Facility (GEF) for funding the development of this research as a part of the "Integrated Solutions for Water, Energy, and Land (ISWEL)" project (GEF Contract Agreement: 6993), and the support of the United Nations Industrial Development Organization (UNIDO). The Water Futures and Solutions Initiative (WFaS) was launched by IIASA, UNESCO/UN-Water, the World Water Council (WWC), the International Water Association (IWA), and the Ministry of Land, Infrastructure and Transport (MOLIT) of the Republic of Korea, and has been supported by the government of Norway, the Asian Development Bank (ADB), and the Austrian Development Agency (ADA). More than 35 organizations contribute to the scientific project team, and an additional 25 organizations are represented in stakeholder groups. Furthermore, WFaS relies on numerous databases compiled and made available by many more organizations, which are referred to in this paper. The research described in this paper would not have been possible without the collaboration of all of these organizations in the WFaS Project Team. The WFaS data are available upon request.

Author Contributions P.G., T.K. and Y.W. designed the study and the associated analysis. P.G. performed all computations. Y.S. preprocessed the data. Y.W., T.K., Y.S., P.B., S.T., 
G.F., R.B. and S.L. designed the water scenarios. P.G, T.K., J.M., T.S. and Y.W. wrote the manuscript. All authors commented on the manuscript.

\section{References}

[1] Nations, U. Transforming Our World: The 2030 Agenda for Sustainable Development. United Nations General Assembly New York (2015).

[2] Vanham, D. et al. Physical water scarcity metrics for monitoring progress towards SDG target 6.4: An evaluation of indicator 6.4.2 "Level of water stress". Science of The Total Environment 613-614, 218-232 (2018).

[3] Wada, Y., Beek, L. P. H. v., Wanders, N. \& Bierkens, M. F. P. Human water consumption intensifies hydrological drought worldwide. Environ. Res. Lett. 8, 034036 (2013).

[4] Wada, Y., van Beek, L. P. H. \& Bierkens, M. F. P. Modelling global water stress of the recent past: on the relative importance of trends in water demand and climate variability. Hydrol. Earth Syst. Sci. 15, 3785-3808 (2011).

[5] Schewe, J. et al. Multimodel assessment of water scarcity under climate change. Proc Natl Acad Sci U S A 111, 3245-3250 (2014).

[6] Greve, P. \& Seneviratne, S. I. Assessment of future changes in water availability and aridity. Geophysical Research Letters 42, 5493-5499 (2015).

[7] Huang, J., Yu, H., Dai, A., Wei, Y. \& Kang, L. Drylands face potential threat under 2 C global warming target. Nature Climate Change 7, 417-422 (2017).

[8] Gudmundsson, L., Seneviratne, S. I. \& Zhang, X. Anthropogenic climate change detected in European renewable freshwater resources. Nature Clim. Change 7, 813-816 (2017).

[9] Qureshi, M. E. \& Whitten, S. M. Regional impact of climate variability and adaptation options in the southern Murray-Darling Basin, Australia. Water Resources and Economics 5, 67-84 (2014).

[10] Flörke, M., Schneider, C. \& McDonald, R. I. Water competition between cities and agriculture driven by climate change and urban growth. Nature Sustainability 1, 51-58 (2018).

[11] Hanasaki, N. et al. A global water scarcity assessment under Shared Socio-economic Pathways - Part 1: Water use. Hydrol. Earth Syst. Sci. 17, 2375-2391 (2013).

[12] Hanasaki, N. et al. A global water scarcity assessment under Shared Socio-economic Pathways - Part 2: Water availability and scarcity. Hydrol. Earth Syst. Sci. 17, 23932413 (2013). 
[13] Mekonnen, M. M. \& Hoekstra, A. Y. Four billion people facing severe water scarcity. Science Advances 2, e1500323 (2016).

[14] Wada, Y. et al. Modeling global water use for the 21st century: the Water Futures and Solutions (WFaS) initiative and its approaches. Geosci. Model Dev. 9, 175-222 (2016).

[15] Hoekstra, A. Y. Water scarcity challenges to business. Nature Clim. Change 4, 318-320 (2014).

[16] Wada, Y., Gleeson, T. \& Esnault, L. Wedge approach to water stress. Nature Geosci 7, 615-617 (2014).

[17] Kahil, M. T., Dinar, A. \& Albiac, J. Modeling water scarcity and droughts for policy adaptation to climate change in arid and semiarid regions. Journal of Hydrology 522, 95 - 109 (2015).

[18] Tortajada, C. Water Management in Singapore. International Journal of Water Resources Development 22, 227-240 (2006).

[19] Devineni, N., Perveen, S. \& Lall, U. Assessing chronic and climate-induced water risk through spatially distributed cumulative deficit measures: A new picture of water sustainability in India. Water Resources Research 49, 2135-2145 (2013).

[20] Winemiller, K. O. et al. Balancing hydropower and biodiversity in the Amazon, Congo, and Mekong. Science 351, 128-129 (2016).

[21] Läderach, P. et al. Climate change adaptation of coffee production in space and time. Climatic Change 141, 47-62 (2017).

[22] Hadarits, M. et al. The interplay between incremental, transitional, and transformational adaptation: a case study of Canadian agriculture. Reg Environ Change 17, 1515-1525 (2017).

[23] Moss, R. H. et al. The next generation of scenarios for climate change research and assessment. Nature 463, 747-756 (2010).

[24] O’Neill, B. C. et al. A new scenario framework for climate change research: the concept of shared socioeconomic pathways. Climatic Change 122, 387-400 (2014).

[25] Dalin, C., Wada, Y., Kastner, T. \& Puma, M. J. Groundwater depletion embedded in international food trade. Nature 543, 700-704 (2017).

[26] Pastor, A. V., Ludwig, F., Biemans, H., Hoff, H. \& Kabat, P. Accounting for environmental flow requirements in global water assessments. Hydrol. Earth Syst. Sci. 18, 5041-5059 (2014). 
[27] Taylor, K., Stouffer, R. \& Meehl, G. An Overview of CMIP5 and the Experiment Design. Bull. Amer. Meteor. Soc. 93, 485-498 (2012).

[28] Perveen, S. \& James, L. A. Multiscale Effects on Spatial Variability Metrics in Global Water Resources Data. Water Resour Manage 24, 1903-1924 (2010).

[29] Perveen, S. \& James, L. A. Scale invariance of water stress and scarcity indicators: Facilitating cross-scale comparisons of water resources vulnerability. Applied Geography 31, 321-328 (2011).

[30] Raskin, P., Gleick, P. \& Institute, S. E. Water Futures: Assessment of Long-range Patterns and Problems. Comprehensive assessment freshwater resources of the world (Stockholm Environment Institute, 1997).

[31] Alcamo, J. et al. Global estimates of water withdrawals and availability under current and future "business-as-usual" conditions. Hydrological Sciences Journal 48, 339-348 (2003).

[32] Water scarcity assessments in the past, present, and future. Earth's Future 5 (2017).

[33] Hawkins, E. \& Sutton, R. The Potential to Narrow Uncertainty in Regional Climate Predictions. Bulletin of the American Meteorological Society 90, 1095-1107 (2009).

[34] Hoekstra, A. Y., Mekonnen, M. M., Chapagain, A. K., Mathews, R. E. \& Richter, B. D. Global Monthly Water Scarcity: Blue Water Footprints versus Blue Water Availability. PLoS ONE 7, e32688 (2012).

[35] Veldkamp, T. I. E. et al. Changing mechanism of global water scarcity events: Impacts of socioeconomic changes and inter-annual hydro-climatic variability. Global Environmental Change 32, 18-29 (2015).

[36] Kates, R. W., Travis, W. R. \& Wilbanks, T. J. Transformational adaptation when incremental adaptations to climate change are insufficient. Proceedings of the National Academy of Sciences 109, 7156-7161 (2012).

[37] Hall, J. W. et al. Coping with the curse of freshwater variability. Science 346, 429-430 (2014).

[38] Kahil, M. T., Connor, J. D. \& Albiac, J. Efficient water management policies for irrigation adaptation to climate change in Southern Europe. Ecological Economics 120, 226-233 (2015).

[39] Leclère, D., Jayet, P.-A. \& de Noblet-Ducoudré, N. Farm-level Autonomous Adaptation of European Agricultural Supply to Climate Change. Ecological Economics 87, 1-14 (2013).

[40] Dittrich, R., Wreford, A. \& Moran, D. A survey of decision-making approaches for climate change adaptation: Are robust methods the way forward? Ecological Economics 122, 79-89 (2016). 
[41] IPCC. Climate Change 2014: Synthesis Report. Contribution of Working Groups I, II and III to the Fifth Assessment Report of the Intergovernmental Panel on Climate Change [Core Writing Team, R.K. Pachauri and L.A. Meyer (eds.)] (IPCC, Geneva, Switzerland, 2014).

[42] Park, S. E. et al. Informing adaptation responses to climate change through theories of transformation. Global Environmental Change 22, 115-126 (2012).

[43] Wheeler, S., Zuo, A. \& Bjornlund, H. Farmers' climate change beliefs and adaptation strategies for a water scarce future in Australia. Global Environmental Change 23, 537$547(2013)$.

[44] OECD. Water, growth and finance - Policy perspectives. (OECD, Paris, 2016).

[45] Grafton, R. Q., Libecap, G., McGlennon, S., Landry, C. \& O’Brien, B. An Integrated Assessment of Water Markets: A Cross-Country Comparison. Rev Environ Econ Policy 5, 219-239 (2011).

[46] Connor, J. D. \& Kaczan, D. Principles for Economically Efficient and Environmentally Sustainable Water Markets: The Australian Experience. In Drought in Arid and Semi-Arid Regions, 357-374 (Springer, Dordrecht, 2013).

[47] Qureshi, M. E., Schwabe, K., Connor, J. \& Kirby, M. Environmental water incentive policy and return flows. Water Resources Research 46 (2010).

[48] El-Sadek, A. Virtual Water Trade as a Solution for Water Scarcity in Egypt. Water Resour Manage 24, 2437-2448 (2010).

[49] Porkka, M., Guillaume, J. H. A., Siebert, S., Schaphoff, S. \& Kummu, M. The use of food imports to overcome local limits to growth. Earth's Future 5, 393-407 (2017).

[50] Grafton, R. Q. et al. Global insights into water resources, climate change and governance. Nature Clim. Change 3, 315-321 (2013).

[51] van Beek, L. P. H., Wada, Y. \& Bierkens, M. F. P. Global monthly water stress: 1. Water balance and water availability. Water Resour. Res. 47, W07517 (2011).

[52] Wada, Y., Wisser, D. \& Bierkens, M. F. P. Global modeling of withdrawal, allocation and consumptive use of surface water and groundwater resources. Earth Syst. Dynam. 5, 15-40 (2014).

[53] Hanasaki, N. et al. An integrated model for the assessment of global water resources Part 1: Model description and input meteorological forcing. Hydrol. Earth Syst. Sci. 12, 1007-1025 (2008). 
[54] Flörke, M. et al. Domestic and industrial water uses of the past 60 years as a mirror of socio-economic development: A global simulation study. Global Environmental Change 23, 144-156 (2013).

[55] Müller Schmied, H. et al. Sensitivity of simulated global-scale freshwater fluxes and storages to input data, hydrological model structure, human water use and calibration. Hydrol. Earth Syst. Sci. 18, 3511-3538 (2014).

[56] Warszawski, L. et al. The Inter-Sectoral Impact Model Intercomparison Project (ISI-MIP): Project framework. PNAS 111, 3228-3232 (2014). 

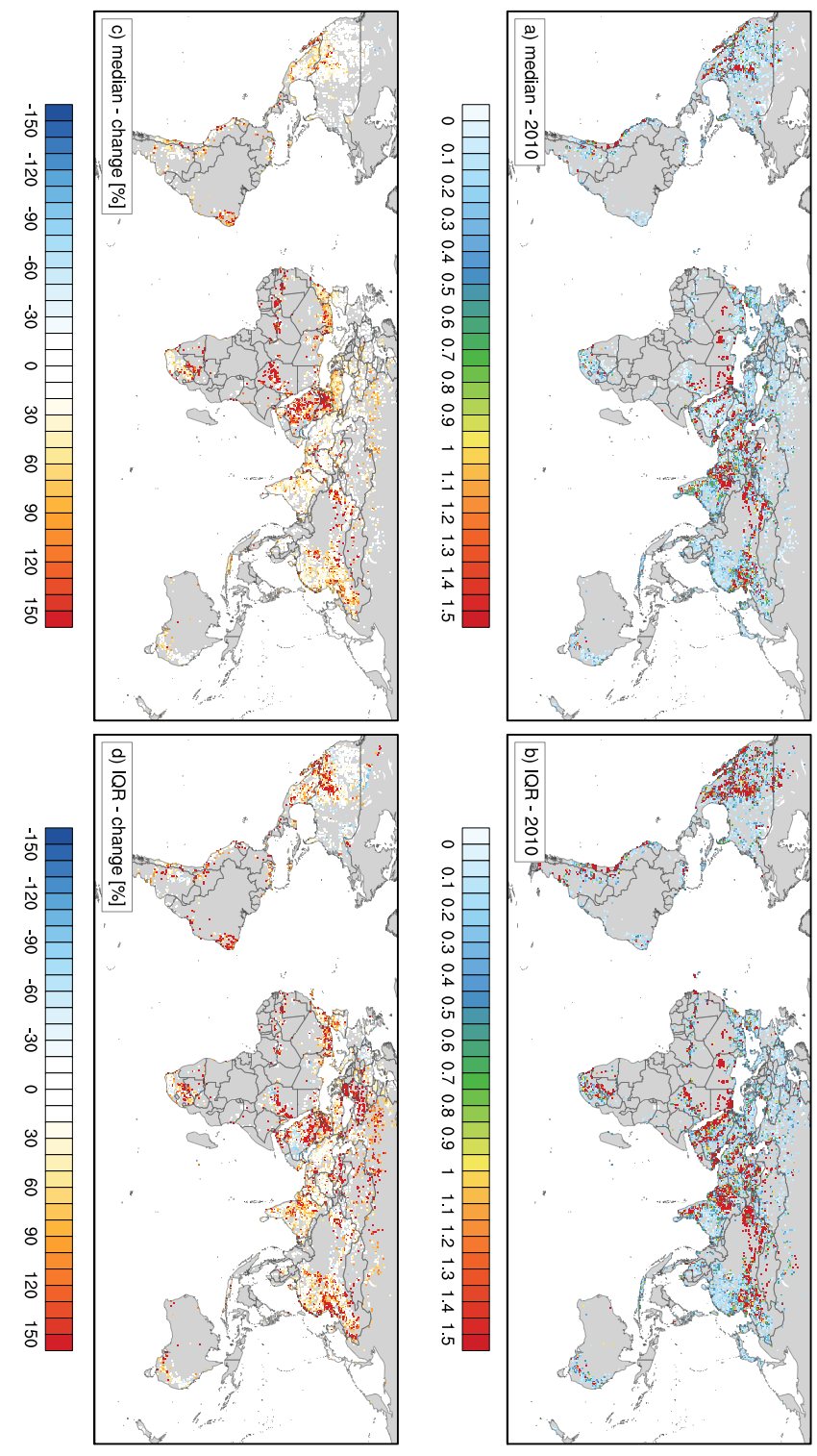

Figure 1: Median and interquartile range derived from the multi-model, multiscenario ensemble of 45 global, decadal water scarcity projections. Median (left column) and interquartile ranges (right column) of the WSI for the first (2006-2015, top row) of the study period. All grid points with the 75th quantile of the WSI being below 0.1 at all time steps are considered as non-water scarce and are masked. Grid points with very low average water demand are also masked (please refer to Supplementary Figure 1 for unmasked results). Relative changes [\%] in the median and IQR of the WSI between the first (2006-2015) and the last decade (2046-2055) of the study period are displayed in the bottom row. 


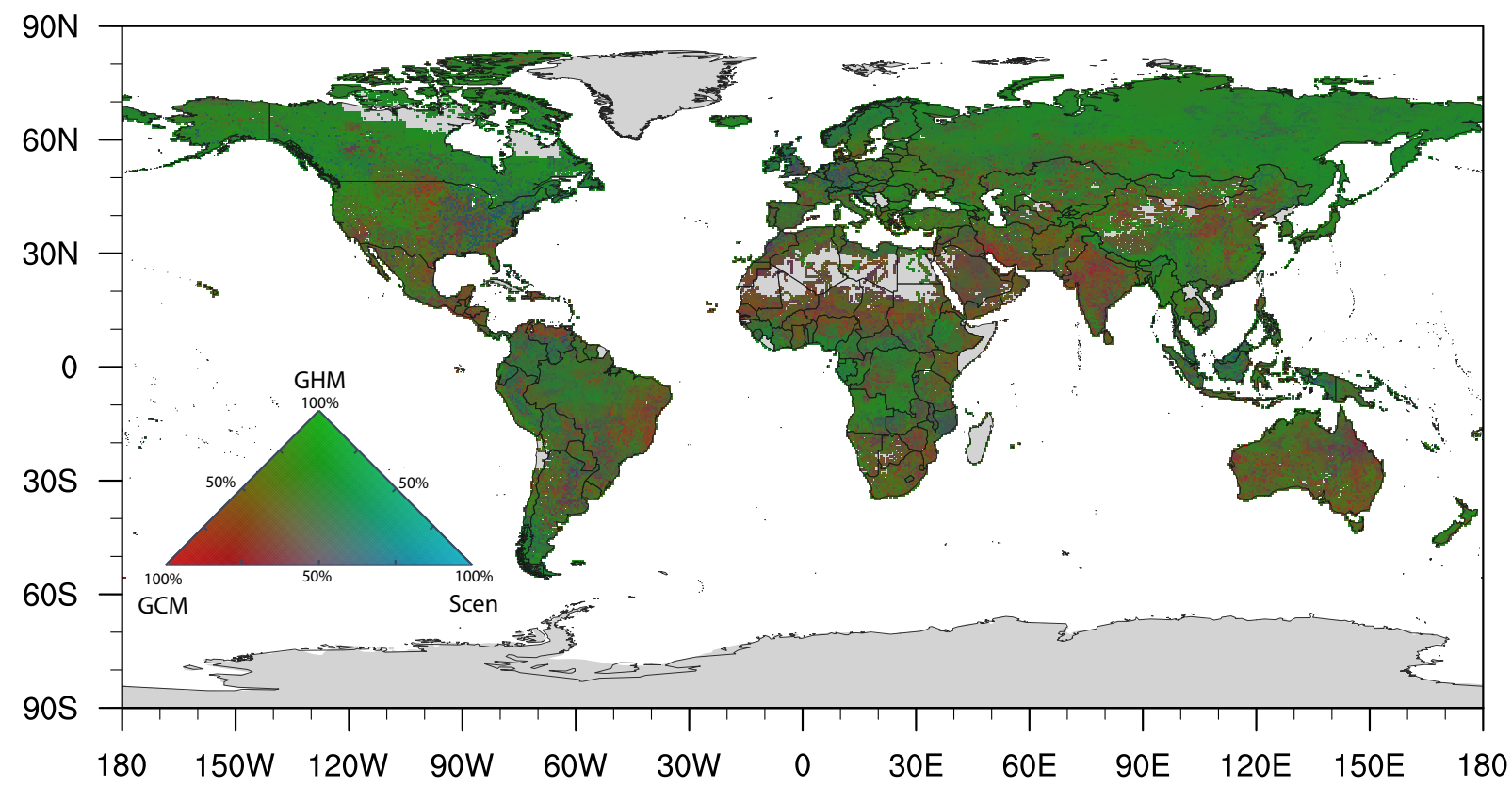

Figure 2: Relative importance of each source of uncertainty within the 2046-2055 period. At each gridpoint, the fractional uncertainty of each source of uncertainty is shown. Please note that the total sum of fractional uncertainties is always $100 \%$. Gray areas denote regions where not all of the 45 ensemble members provide data. Absolute values of the IQR are provided in Fig. 1d. 

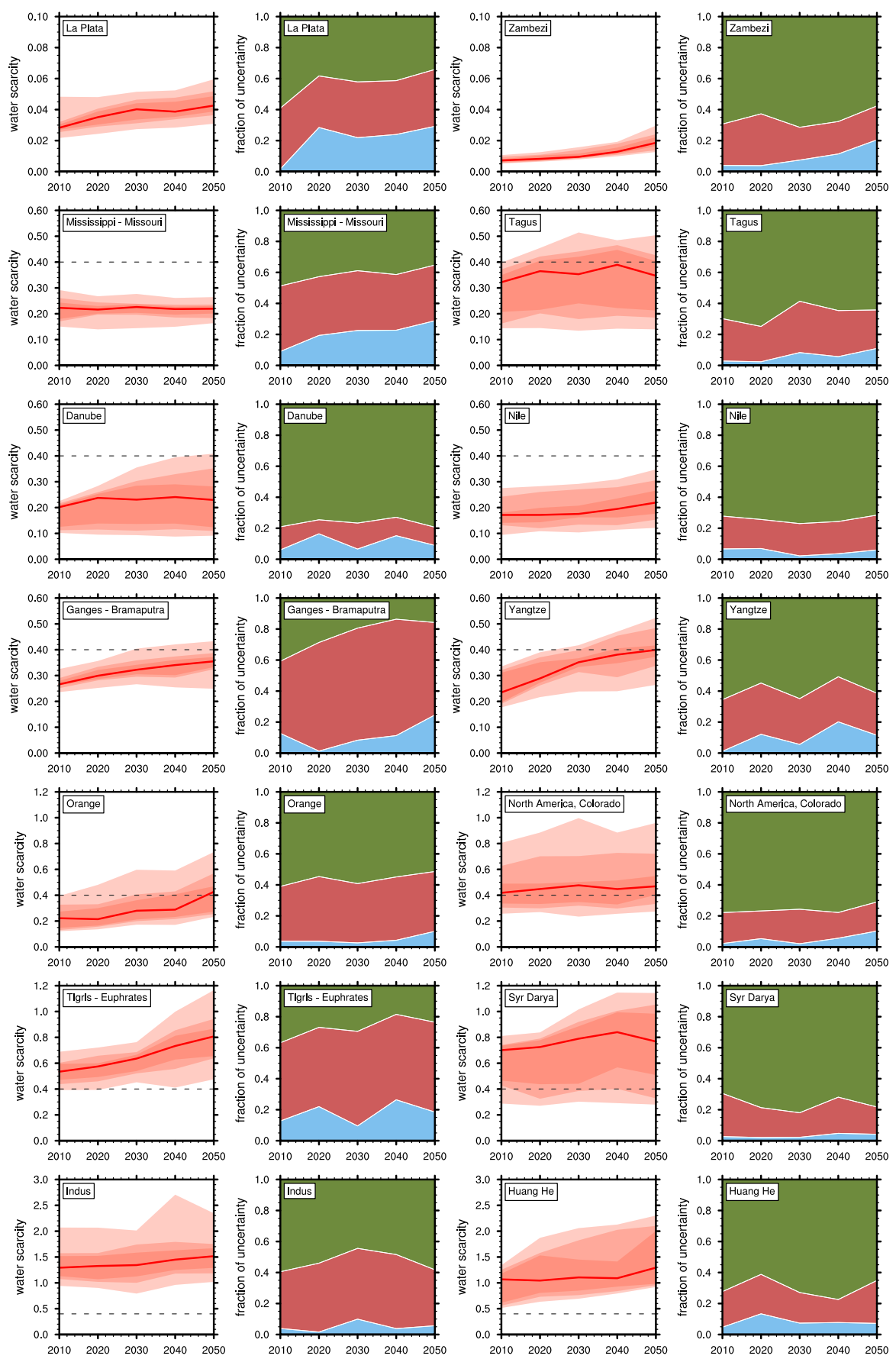

Figure 3: Basin averages of decade-to-decade change of the uncertainty distribution and the relative importance of different uncertainty sources. Plots in the first and third column illustrate the uncertainty range by showing time series of chosen quantiles and the median for a subset of 14 major river basins worldwide. Please note the different scales of the two uppermost plots and the bottom plots. The dashed line indicates WSI $=0.4$. Plots in the second and fourth column represent the time-evolution of the relative importance of each driver of uncertainty within the same subset of major river basins (green: GHMs, red: GCMs, blue: scenario). 


\section{Policy challenges}

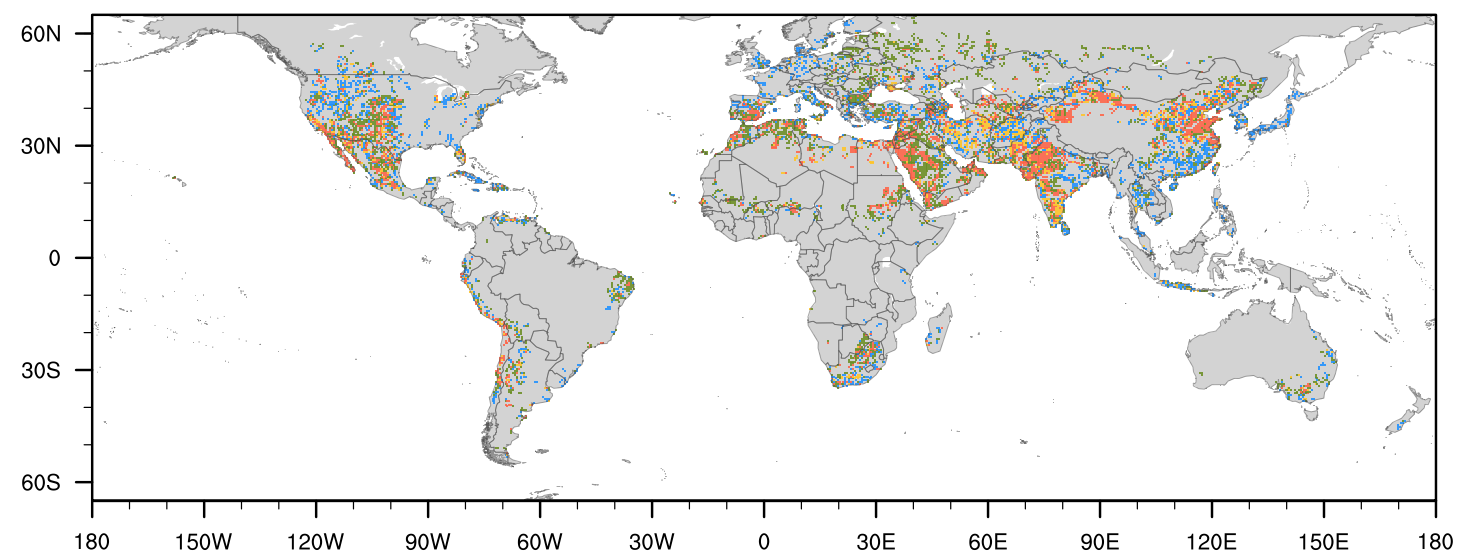

\begin{tabular}{|c|c|c|c|c|}
\hline Challenges & low & ium & medium & high \\
\hline $\begin{array}{l}-------- \\
\text { uncertainty (2010) } \\
-------- \\
\text { uncertainty } \\
\text { changes }(2010-50)\end{array}$ & \begin{tabular}{l|l|} 
& 0.4 \\
& \\
\end{tabular} & $\begin{array}{l}\text { < } 0.4 \\
\text { men-, slightly water scarce } \\
\text { increase }\end{array}$ & $\begin{array}{l}\text { severely water scarce } \\
\end{array}$ & $\begin{array}{c}\text { severely water scarce } \\
\text { medium to high } \\
\text { IQR }>0.6 \\
\text { medium to high } \\
\text { increase }\end{array}$ \\
\hline $\begin{array}{l}\text { Policy actions } \\
------- \\
\text { Decision-making } \\
\text { frameworks }\end{array}$ & $\begin{array}{l}\text { monitoring and } \\
\text { reviewing risks } \\
\text { conventional } \\
\text { approaches } \\
\text { such as e.g., } \\
\text { cost benefit analysis }\end{array}$ & $\begin{array}{c}\text { transitional changes } \\
\text { iterative } \\
-------- \\
\text { beginning with } \\
\text { low-regret options }\end{array}$ & $\begin{array}{c}\text { transitional/ } \\
\text { transformational } \\
\text { changes }\end{array}$ & $\begin{array}{c}\text { transformational } \\
\text { changes } \\
-------- \\
\text { ision making } \\
---------- \\
\text { evaluating } \\
\text { transformational } \\
\text { options }\end{array}$ \\
\hline
\end{tabular}

Figure 4: Identifying areas of low, medium and high water policy challenges. Potential areas of differing management challenges with respect to anticipated water scarcity conditions within the first half of the 21st century. Please see Supplementary Figure 2 for zoomed-in map segments of most affected regions. All grid points with the 75th quantile of the WSI being below 0.1 at all time steps are considered as non-water scarce and are masked. Grid points with very low average water demand are also masked (grey). The characteristics of projected water scarcity changes and generic policy implications (as presented in Sec. 3.2) are summarized in the table below. The graphs in the second row of the table represent the time-evolution of the IQR of the respective median IQR of the multi-model ensemble at all grid points within the particular challenge area as displayed in the map (please mind the differences in the y-axis scale between the first and last two graphs). 